\title{
Improved Mechanical Properties of PVA-Chitosan Polymeric Porous Scaffolds for Tissue Engineering
}

\author{
Md. Shariful Islam ${ }^{1,2, *}$, Mitsugu Todo ${ }^{1}$ \\ ${ }^{1}$ Research Institute for Applied Mechanics, Kyushu University, Fukuoka, Japan \\ ${ }^{2}$ Department of Animal Husbandry and Veterinary Science, University of Rajshahi, Rajshahi, Bangladesh
}

Email address:

surus06@yahoo.com (Md. S. Islam), todo@riam.kyushu-u.ac.jp (M. Todo)

\section{To cite this article:}

Md. Shariful Islam, Mitsugu Todo. Improved Mechanical Properties of PVA-Chitosan Polymeric Porous Scaffolds for Tissue Engineering. American Journal of Clinical and Experimental Medicine. Vol. 3, No. 5, 2015, pp. 268-274. doi: 10.11648/ j.ajcem.20150305.22

\begin{abstract}
Highly porous HA scaffolds were synthesized from bioceramics by using the polyurethane (PU) sponge template method. The as-prepared HA scaffolds were then fabricated with poly (vinyl alcohol)/chitosan (PVA/CS) and collagen/chitosan $(\mathrm{COL} / \mathrm{CS})$ polymeric materials at 4:1 ratio in coating and 2-phase atmospheric condition. Further, the porous microstructure of fabricated biomaterials were characterized by FE-SEM and mechanical properties were tested by using Shimadzu Compact Tabletop Testing Machine EZTest. It was revealed from the study that incorporation of PVA/CS or COL/CS polymeric materials into pure HA scaffolds either coating or 2-phase condition enhanced the mechanical properties of fabricated biomaterials significantly. Biocompatibility of fabricated biomaterials were checked by culturing Human Mesenchymal Stem cell (hMSC) for a period of 7 days over the prepared scaffolds and it was found that hMSC responded well and grown excellently over the all specimens of fabricated scaffolds. Finally, the results revealed that maximum stress value $(0.77 \mathrm{MPa})$ was obtained from HA-PVA/CS 2-phase with cell samples of 7 days culture and followed by HA-PVA/CS coating with cell $(0.75 \mathrm{MPa})$ due to formation of extra cellular matrix (ECM) reinforcement which allowed specimens undergo densification and stress continued to increase.
\end{abstract}

Keywords: Porous Scaffolds, Mechanical Properties, Biocompatibility, Tissue Engineering, Human Mesenchymal Stem cell (hMSC)

\section{Introduction}

In recent years new technologies have enabled the in vitro growth of many different cell types. Tissue engineering is one such aspect that utilizes both Engineering and life science discipline to either maintain existing tissue structure or to enable tissue growth [1]. Scaffolds act as a substrate to promote cell adhesion, maintenance of differentiated function without hindering proliferation, template to organize and direct the growth of cell and help in the formation of extra cellular matrix (ECM) [2]. A biodegradable scaffold in bone tissue engineering serves as a temporary skeleton inserted into the sites of defective or lost bone to support and stimulate bone tissue regeneration while it gradually degrades and is replaced by new bone tissue [3-5]. Both bioactive ceramics and polymers have been developed and analyzed for use as tissue engineering scaffolds. Bioactive ceramics have chemical composition resembling that of natural bone, allow osteogenesis to occur, and can provide a bony contact or bonds with host bone $[6,7]$. Despite their favorable biological properties, bioceramics are inherently brittle and have low biodegradation rates, which severely limits their clinical use [8]. Bioceramics, hydroxyapatite (HA) and $\beta$-tricalcium phosphate $(\beta$-TCP) for instant, has been introduced to bone tissue engineering for their unique properties of bioactivity, biocompatibility and osteoconductivity $[9,10]$. HA has been introduced in bone tissue engineering as a scaffold material mainly due to its unique properties such as excellent bioactivity, biocompatibility, osteoconductivity and non-toxicity [9-12]. In addition to these properties, it is a major inorganic component of bone and has been used as both filler and porous bone scaffolds to enhance mechanical properties, bone ingrowth and osseointergration [12-14]. However, low strength and brittleness of HA has limited its wide applications in hard tissue implant [15]. To improve the extensive range of applications and use effectively in load bearing compartments, the mechanical properties of the HA porous scaffold should be enhanced [16]. 
The scaffold can be developed using either natural or synthetic polymers. Nowadays, natural polymers are widely used for organ regeneration since they facilitate cell attachment and maintenance of differentiation function [17]. Collagen $(\mathrm{COL})$ is one of the natural biopolymer and provides good biocompatibility and low antigenicity as well as it responds well to cell attachment and proliferation [18]. Chitosan (CS) is derived from chitin, is also a natural polymer and has recently attracted increasing interest both in research and development aspects. Whereas, Poly (vinyl alcohol) (PVA) is typically non-toxic and water-soluble synthetic polymer. It is also biocompatible, can be degradable by method and yields comparably high fiber forming. According to compatible properties to $\mathrm{CS}$, it is believed that the composite of PVA and $\mathrm{CS}$, can lead to the novel functional biocompatible materials for specific biomedical applications [19]. For successful tissue engineering of cartilage, a mechanically robust and biocompatible biomaterial is needed on which MSCs undergo chondrogenic differentiation and form 3-dimensional cartilage-like tissue [20]. Porosity and interconnectivity are important for an accurate diffusion of nutrients and gases and for the removal of metabolic waste resulting from the activity of the cells proliferated into the scaffold [21]. Then the selection of the most appropriate materials to produce a scaffold to be applied in bone tissue engineering applications is very important because its properties will provide great potential properties of the scaffold [22].

Hence this study was set out to develop a biomimetic scaffold by incorporating osteoconductive HA materials into PVA to produce a highly porous and extremely biocompatible scaffolds. Further, as-prepared HA scaffolds were retreated with biocompatible COL, PVA and CS to enhance the mechanical properties of prepared scaffolds as well as to improve osteogenic performance within our laboratory.

\section{Materials and Methods}

\subsection{Scaffold Development}

HA porous materials were fabricated by using the polyurethane (PU) sponge template method. HA slurry was prepared from commercial micro-HA powder (PS-1, Sangi Co, Ltd.) containing particle size of 0.03 to $0.1 \mu \mathrm{m}$ mixed with poly vinyl alcohol (PVA: $(\mathrm{CH} 2 \mathrm{CHOH}) n)(165-17915$, $\mathrm{Mw}=1500-1800 \mathrm{~g} / \mathrm{mol}$, Wako Pure Chemical Industries, Ltd.) $5 \mathrm{wt} \%$ solution following the ratio of 1:1 (1g HA mix with $1 \mathrm{ml}$ PVA solution) using a centrifuge mixing machine (Imoto Co, Ltd.) with 8 min kneading and 4 min degassing (removing gas-bubbles). PU sponge templates (HR-30, Bridgestone) cut into $1 \times 1 \times 1 \mathrm{~cm}$ dimensions were immersed into the slurry.

The slurry-immersed PU sponge templates were then fully compressed $(95 \%)$ by employing two plates to remove excess slurry. The slurry-immersed templates were dried at $60^{\circ} \mathrm{C}$ for 24 hours and then the process of calcination was performed at $400^{\circ} \mathrm{C}$ for 6 hours with a heating rate of $10^{\circ} \mathrm{C} / \mathrm{min}$, followed by the sintering process for 3 hours at $1300^{\circ} \mathrm{C}$.

\subsection{Fabrication of HA Porous Scaffolds with COL, PVA and $C S$}

The as-prepared HA porous scaffolds were fabricated with COL, PVA and CS in two ways, a) coating and b) 2-phase. At first $\mathrm{COL} / \mathrm{CS}$ or PVA/CS mixed solution were prepared at $4: 1$ ratio and were dissolved in $2 \%$ acetic acid at $5 \mathrm{wt} \%$. The solution was magnetically stirred at room temperature for 2 hours. For type (a), as-prepared HA porous scaffolds were dipped into the PVA/CS or COL/CS solution and vacuumed. Excess solution was taken out and dried at room temperature for at least 48 hours. These samples were named as 'HA-PVA/CS or HA-COL/CS coated scaffolds'. On the other hand, for type (b) both types of coated HA scaffolds were fabricated in a specific freeze-drying procedure. HA-PVA/CS or HA-COL/CS coated scaffolds were frozen at $-20^{\circ} \mathrm{C}$ for 24 hours and then freeze-dried at $-50^{\circ} \mathrm{C}$ for 24 hours. These specimens were named as 'HA-PVA/CS or HA-COL/CS 2-phase scaffolds'.

\subsection{Microstructural Characterization}

FE-SEM is one of the most versatile material morphology characterization techniques available. Specimen microstructures morphology was carried out using FE-SEM (Hitachi, Ltd. S-4100) according to Islam et al [23]. To make specimens become conductive for being analyzed by FESEM, specimens were placed on the specimen holder attached by using carbon tape and adding electro-conductive dotite D-550 (Fujikura Kasei Co, Ltd.) at the bottom of the sample. Then specimens were coated with a thin layer of $\mathrm{Pt} / \mathrm{Pd}$-alloy using vacuum sputter (Hitachi E1030 Ion Sputter).

\subsection{Compression Testing}

Specimens obtained after fabrication process were subjected to study mechanical properties. Specimen's dimensions of $\mathrm{L}(\mathrm{mm})$ in length, $\mathrm{W}(\mathrm{mm})$ in width and $\mathrm{H}$ $(\mathrm{mm})$ in height were measured prior to the test due to shrinkage after sintering. Compression tests were performed by using Shimadzu Compact Tabletop Testing Machine EZTest (EZ-S Series) equipped with $500 \mathrm{~N}$ load cell and a crosshead speed of $1 \mathrm{~mm} / \mathrm{min}$. Force $\mathrm{F}(\mathrm{N})$ and displacement $\Delta \mathrm{H}(\mathrm{mm})$ were determined and then stress $\sigma(\mathrm{MPa})$ (Equation 1) and strain $\epsilon$ (Equation 2) were calculated. Elastic modulus E was acquired from the initial slope of the stress strain curve before the failure of specimen. For each condition, 6 samples were tested and average value was used.

$$
\begin{aligned}
& \text { Stress } \sigma=\frac{F}{L \times W} \\
& \text { Strain } \in=\frac{\Delta H}{H}
\end{aligned}
$$

\subsection{Equilibrium Swelling Analysis}

Swelling behavior of each types of scaffold sintering at $1300^{\circ} \mathrm{C}$ was evaluated according to Sang et al [24]. Simply, scaffolds were immersed in PBS ( $\mathrm{pH}=7.4)$ solution for 1,3 , 24 , and $48 \mathrm{~h}$ at $37{ }^{\circ} \mathrm{C}$ in a humidified atmosphere of $5 \%$ 
CO2 described by Arahira and Todo [25]. The influence of alkaline $\mathrm{pH}$ on swelling behavior of the scaffold was determined by carrying out studies using $0.05 \mathrm{M}$ phosphate buffer ( $\mathrm{pH}$ 7.4), until equilibrium was obtained. At first dry weight of each sample was recorded for determining the equilibrium swelling characteristics and then swollen weights of various scaffolds were accurately determined after removing the water adsorbed by them using filter paper. Finally, the swelling ratio was evaluated using the following formula [24].

$$
\text { Swelling ratio }=\frac{\text { Wwet-Wdry }}{\text { Wdry }}
$$

Where $\mathrm{W}_{\text {dry }}$ is the initial weight of the dried scaffold and $\mathrm{W}_{\text {wet }}$ is the weight of the scaffold with soaked in PBS.

\subsection{Cell Experiment}

Human Mesenchymal Stem cell (hMSC) (UE6E7T-3, Riken Bioresource Center) were cultivated in $5 \mathrm{ml}$ of MEM $\alpha$ (Wako Pure Chemical Industries, Ltd.) supplemented with 10\% of fetal bovine serum (FBS) (Gibco) and $1 \%$ of penicillin-streptomycin (Pen-Strep) (Sigma Life Science). Cells were incubated under the standard cell culture condition at $37^{\circ} \mathrm{C}$ in an atmosphere of $5 \% \mathrm{CO} 2$ and $70 \%$ humidity. The medium was changed twice per week. When cells reached sub-confluence, they were harvested with trypsin (Gibco) and sub-cultured. All the experiments were performed under the identical condition.

hMSCs were cultured over the developed HA-PVA/CS or HA-COL/CS scaffolds. The Scaffolds of uniform size were placed in sterile culture plates and were sterilized overnight using UV radiation, under laminar airflow chamber. These sterilized scaffolds were then transferred to three $6 \mathrm{~mm}$ well-sterilized plastic culture plates individually and were labelled sample Nos. 1, 2 and 3. One $\mathrm{ml}$ of cell suspension (containing $7.4 \times 10^{4}$ cells) was added to sample Nos. $1 \& 2$ (test samples) and the third sample was kept as control. The test samples Nos. $1 \& 2$ were cultured with $2 \mathrm{ml}$ of MEM $\alpha$ supplemented with $10 \%$ of FBS and $1 \%$ of penicillin-streptomycin for a week. The control sample was also supplemented with $2 \mathrm{ml}$ of MEM $\alpha$ growth media. The medium was changed twice in every week. Optical photographs of the cultured hMSCs were taken at 7 days of culture. The images were taken using an Olympus inverted CKX41microscope with a numerical light field condenser (N.A.0.3), which delivers a very narrow beam of white light from tungsten lamp $(6 \mathrm{~V}, 30 \mathrm{Whalogen}$ illumination) on top of the sample. A $20 \times$ objective was used to collect the sample images.

\subsection{Statistical Analysis}

All data were presented as means \pm standard deviation (SD) and derived from 2-6 independent samples. The analysis of variance (ANOVA) was used to evaluate the recorded data by and any difference was considered statistically significant when the $p$ value was $<0.05$.

\section{Results and Discussion}

\subsection{Microstructural Morphology of Scaffolds}

The SEM micrographs of the pure $\mathrm{HA}$ and fabricated HA-PVA/CS or HA-COL/CS scaffolds are shown in Fig. 1. In the Fig.1 pure HA and fabricated porous scaffolds; HA-PVA/CS coating, HA-COL/CS coating, HA-PVA/CS 2-phase and HA-COL/CS 2-phase are represented by (a), (b), (c), (d) and (e), respectively. All the scaffolds shown very similar porous structure; however, the fabricated scaffold had more straight aligned walls than the pure HA scaffold, indicating the increased stiffness by distribution of PVA/CS or COL/CS over the pure HA materials. For a porous material, porosity is a critical property to consider. Porosity of specimen was determined based on the density of HA porous material and the density of HA and expressed in percentage as shown in Equation (4). The density of specimen was calculated based on weight with volume of HA porous material and density of $\mathrm{HA}$ is $3 \cdot 16 \mathrm{~g} / \mathrm{cm}^{3}[26,27]$.

Porosity of HA porous material $[\%]=\frac{\rho \mathrm{HA}-\rho \text { porous } \mathrm{HA}}{\rho \mathrm{HA}} \times 100$

Where, $\rho$ HA is the density of HA and $\rho$ porous HA is the density of HA porous material.

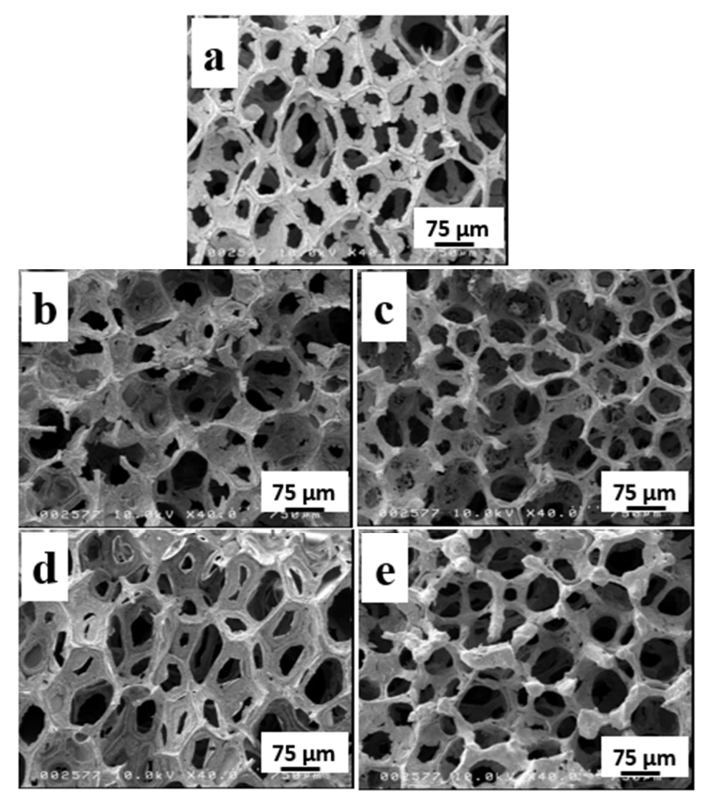

Figure 1. SEM micrographs of pure $H A$ and fabricated scaffolds; (a) pure $H A$, (b) HA-PVA/CS coating, (c) HA-COL/CS coating, (d) HA-PVA/CS 2-phase and (e) HA-COL/CS 2-phase scaffold.

The porosity of the pure HA scaffold evaluated from Eq. 4 was about $94.8 \%$, while that of the fabricated HA-PVA/CS coating, HA-COL/CS coating, HA-PVA/CS 2-phase and HA-COL/CS 2-phase scaffolds were 93.5\%, 92.3\%, 92.6\% and $89.5 \%$, respectively, indicating that the addition of $\mathrm{PVA} / \mathrm{CS}$ or COL/CS polymeric materials slightly reduced the porosity of the pure HA scaffold (Table. 1). Further, 2-phase condition also slightly reduced porosity of the fabricated scaffolds than that of coating one. 
Table 1. Porosity of pure HA and fabricated scaffolds.

\begin{tabular}{ll}
\hline Scaffolds type & Porosity $\mathbf{( \% )}$ \\
\hline Pure HA & 94.8 \\
HA-PVA/CS coating & 93.5 \\
HA-COL/CS coating & 92.3 \\
HA-PVA/CS 2-phase & 92.6 \\
HA-COL/CS 2-phase & 89.5 \\
\hline
\end{tabular}

\subsection{Compressive Mechanical Properties}

The compressive mechanical properties were measured in terms of modulus, fracture stress, strain energy density and

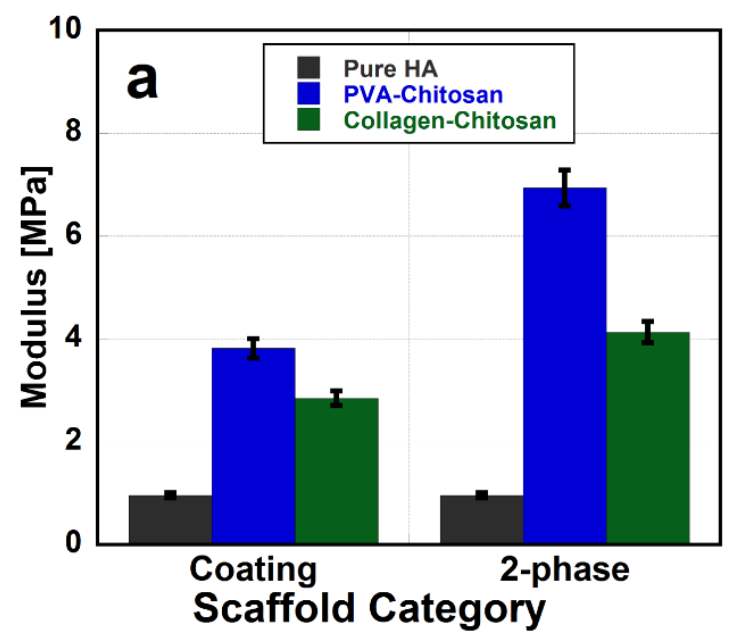

Figure 2. Modulus (a) and fracture stress (b) of pure HA and fabricated HA-COL/CS or HA-PVA/CS scaffolds.

Figure 3. shown the strain energy density of pure HA and fabricated HA-PVA/CS or HA-COL/CS scaffolds of both categories. It was observed that strain energy density of HA-PVA/CS coating and 2-phase scaffolds were 19.71 and $48.93 \mathrm{KJ} / \mathrm{m}^{3}$, respectively, whereas for HA-COL/CS coating and 2-phase scaffolds it was 4.35 and $3.22 \mathrm{KJ} / \mathrm{m}^{3}$, respectively.



Figure 3. Strain energy density of pure $H A$ and fabricated $H A-P V A / C S$ or HA-COL/CS scaffolds.

It was clearly evidenced that all the studied compressive mechanical properties were improved by the introduction of strain-stress curve (s-s). The compressive modulus and fracture stress of pure HA and fabricated HA-PVA/CS or HA-COL/CS scaffolds are shown in Fig. 2 (a) and (b). ). It was noticed that modulus of HA-PVA/CS coating and 2-phase scaffolds were 3.81 and $6.94 \mathrm{MPa}$, respectively, whereas for $\mathrm{HA}-\mathrm{COL} / \mathrm{CS}$ coating and 2-phase scaffolds it was 2.85 and $4.13 \mathrm{MPa}$, respectively, but for pure HA scaffolds modulus was only $0.960 \mathrm{MPa}$. Further, fracture stress of HA-PVA/CS coating, HA-PVA/CS 2-phase, HA-COL/CS coating and HA-COL/CS 2-phase scaffolds were $0.38,0.63,0.14$ and $0.15 \mathrm{MPa}$, respectively, whereas, for pure HA scaffolds it was average only $0.015 \mathrm{MPa}$.

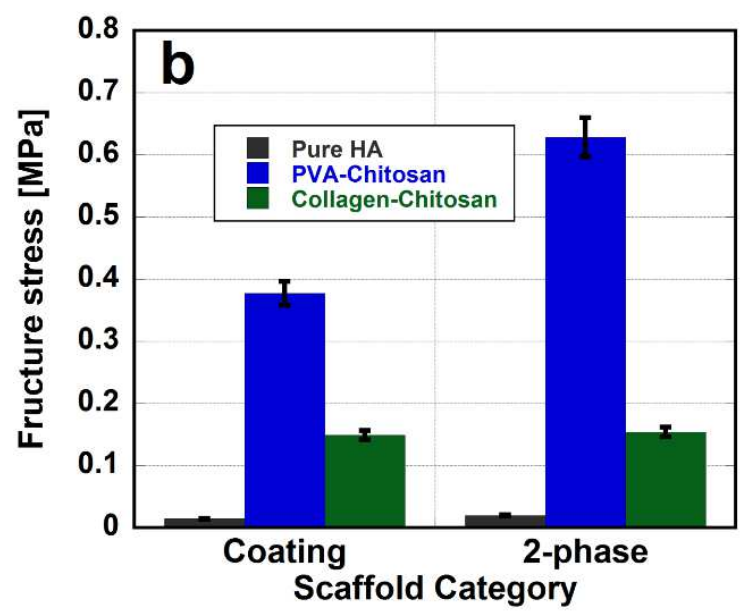

PVA/CS or COL/CS polymeric phase. It was also noted that between the PVA/CS and COL/CS polymeric phase PVA/CS phase had the higher influence in the improvement of mechanical properties. Moreover, the 2-phase condition of the fabricated scaffolds had the maximum influence on all the studied compressive mechanical properties. These results clearly indicated that the compressive mechanical properties can be controlled by incorporating various polymeric materials in to pure HA. Figure 4. (A) and (B) shown typical stress-strain curves under compression of all fabricated scaffolds with or without hMSCs. All specimens showed different pattern of stress-strain curve. Stress increased linearly corresponding to the linear elasticity until the crack initiated. Then after reaching the critical point, stress decreased due to the failure of HA frameworks.

It was observed that stress values for fabricated HA-PVA/CS 2-phase without cell, HA-PVA/CS 2-phase with cell, HA-PVA/CS coating without cell and HA-PVA/CS coating with cell samples of 7 days culture were $0.50,0.77$, 0.47 and $0.75 \mathrm{MPa}$, respectively, whereas, for $\mathrm{HA}-\mathrm{COL} / \mathrm{CS}$ 2-phase without cell, HA-COL/CS 2-phase with cell, HA-COL/CS coating without cell and HA-COL/CS coating with cell samples the values were $0.26,0.48,0.16$ and 0.37 $\mathrm{MPa}$, respectively. So, it was revealed from the results that maximum stress value $(0.77 \mathrm{MPa})$ was obtained from HA-PVA/CS 2-phase with cell samples of 7 days culture and followed by HA-PVA/CS coating with cell $(0.75 \mathrm{MPa})$. 

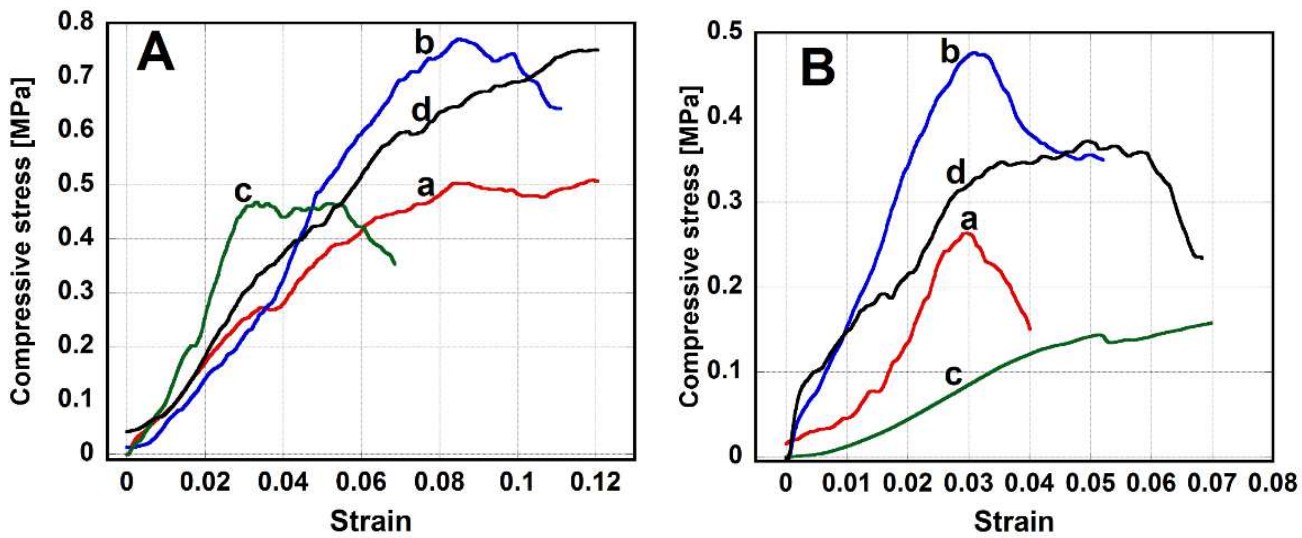

Figure 4. Compressive stress-strain curves of fabricated HA-PVA/CS scaffolds (A) and HA-COL/CS scaffolds (B). In plate (A), HA-PVA/CS 2-phase without cell (a), HA-PVA/CS 2-phase with cell (b), HA-PVA/CS coating without cell (c) and HA-PVA/CS coating with cell (d), whereas, in plate (B), HA-COL/CS 2-phase without cell (a), HA-COL/CS 2-phase with cell (b), HA-COL/CS coating without cell (c) and HA-COL/CS coating with cell (d) were denoted.

Every specimens with hMSC shown better stress performance than their counterparts without hMSC due to EMC reinforcement which allowed specimens undergo densification and stress continued to increase. However, we assumed that at the 7th days of cell seeding the samples showed higher maximum stress than that of the without hMSC specimen due to the increase in ECM layer to strengthen the specimens.

\subsection{Equilibrium Swelling Behavior}

The swelling behavior of each types of scaffold sintering at $1300{ }^{\circ} \mathrm{C}$ shown in Fig. 5. The ability of scaffold to swell plays an important role during the in vitro culture studies.

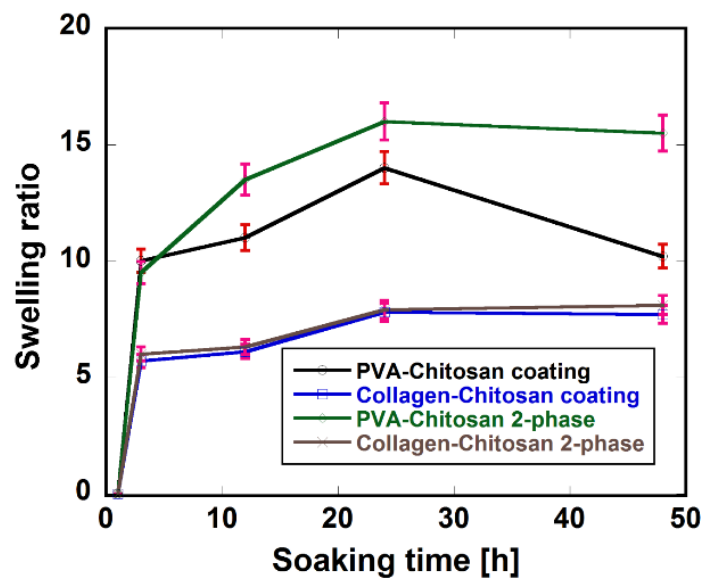

Figure 5. Swelling behavior of as-prepared pure $H A$ and fabricated HA-PVA/CS or HA-COL/CS scaffolds.

Reason for this simple property to consider was when the scaffolds are capable of swelling they allow their pore size to increase in diameter in order to swell thus facilitating the cells not only to just attach but also they allow them to penetrate inside the scaffolds to grow in a three-dimensional fashion during in vitro culture studies. Moreover the cells avail the maximum internal surface area of the scaffold.
From comparative studies of the swelling properties of various scaffolds, the swelling ratio of the fabricated HA-PVA/CS coating, HA-PVA/CS 2-phase, HA-COL/CS coating and $\mathrm{HA}-\mathrm{COL} / \mathrm{CS}$ 2-phase scaffolds reached its maximum of about 14.0, 16.0, 7.8 and 8.0 , respectively at $24 \mathrm{~h}$, and then keep almost constant.

\subsection{Cell Growth}

hMSC were cultured over the scaffold developed from $\mathrm{PVA} / \mathrm{CS}$ and COL/CS polymeric materials in both coating and 2-phase condition. Fig. 6 shown the 7 days culture photographs of various samples.

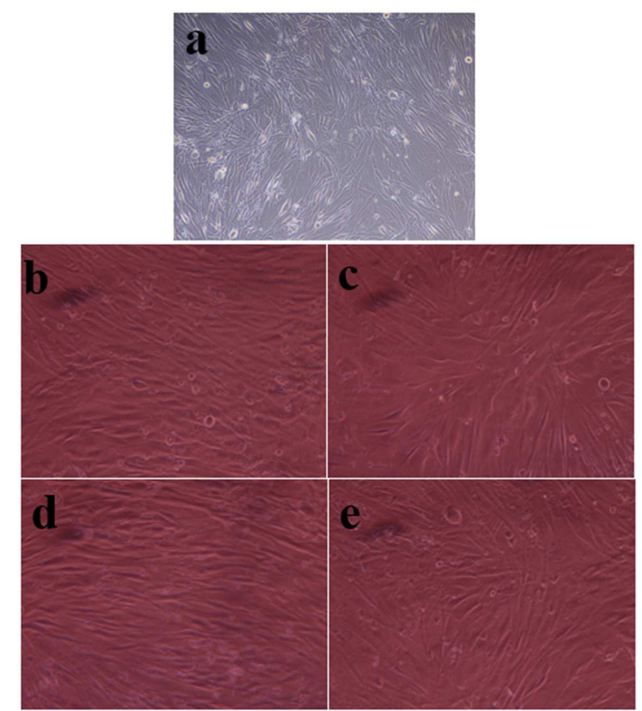

Figure 6. Optical photograph of day 7 culture of hMSC (magnification 20×). Here, hMSC in $25 \mathrm{~cm} 2$ tissue culture flasks, HA-PVA/CS coating, HA-COL/CS coating, HA-PVA/CS 2-phase and HA-COL/CS 2-phase scaffolds were represented by (a), (b), (c), (d) and (e), respectively.

All the samples that contained $1 \mathrm{ml}$ of cell suspension, supplemented with $2 \mathrm{ml}$ of growth media, showed positive attachment and growth. The morphological images of hMSCs were closely investigated at 7 th day of culture and finally 
vital observations were made from optical photographs taken. It was revealed from the photographs that the cells were seeded well over the fabricated scaffolds and started to attach and grown vigorously (Fig. 6). So, it was noteworthy to conclude that the fabricated scaffolds were biocompatible for tissue engineering although other biocompatible parameters and cell differentiation studies are under progress in our laboratory.

\section{Conclusion}

HA porous scaffolds were developed using template method and were further fabricated with natural (COL, CS) or synthetic polymers (PVA). Analytical studies revealed that incorporation of COL, CS and PVA combinedly with coating or 2-phase condition improved the mechanical properties of fabricated biomaterials. Moreover, prepared biomaterials were tested for their biocompatibility with hMSC and it was also found that hMSCs were excellently proliferate over the all sorts of fabricated scaffolds. It was interestingly noted that fabricated HA-PVA/CS 2-phase scaffold was best performer than others. Hopefully, further biocompatible studies will be discussed in our next full research article.

\section{Acknowledgements}

The present work was supported by Grant-in-Aid for Scientific Research (No.26•04362) from Japan Society for the Promotion of Science (JSPS) and Grant-in-Aid for JSPS Fellows (No. P14362).

\section{References}

[1] E. Wintermantel, J. Mayer, J. Blum, K. L. Eckert, P. Luscher, and M. Mathey, "Tissue engineering scaffolds using superstructures” Biomaterials, 1996, vol.17, pp. 83-91.

[2] R. C. Thomson, M. C. Wake, M. J. Yaszemski, and A. G. Mikos, "Biodegradable polymer scaffolds to regenerate organs," Adv. Biopolym., 1997, vol. 122, pp. 245-274.

[3] A. Persidis, "Tissue engineering" Nat. Biotechnol., 1999, vol.17, pp. 508-510.

[4] R. F. Service, "Tissue engineers build new bone," Science, 2000 , vo. 289 , pp. $1498-1500$.

[5] H. Petite, V. Viateau, W. Bensaid, A. Meunier, C. de Pollak, M. Bourguignon, et al., "Tissue engineered bone regeneration. Nat. Biotechnol., 2000, vol. 18, pp. 959-963.

[6] M. Jarcho, "Calcium phosphate ceramics as hard tissue prosthetics", Clin. Orthop. 1981, pp. 259-78.

[7] L. L. Hench, and J. Wilson, "Surface-active biomaterials," Science, 1984, vol. 226, pp. 630-706.

[8] Z. Li, R. H. Ramay, Kip D. Hauch, D. Xiao, and M. Zhang, "Chitosan-alginate hybrid scaffolds for bone tissue engineering,” Biomaterials, 2005, vol. 26, pp. 3919-3928.

[9] I. L. Hench, "Bioceramics: From concept to clinic," J. Am. Ceram. Soc., 1991, vol. 74, pp. 1487-1510.
[10] T. L.T. Kitsugi, T. Yamamuro, T. Nakamura, and M. Oka, "Transmission electron microscopy observations at the interface of bone and four types of calcium phosphate ceramics with different calcium/phosphorus molar ratios" Biomaterials, 1995, vol. 16, pp. 1101-1107.

[11] R. Murugan and S. Ramakrishna, "Production of ultra-fine bioresorbable carbonated hydroxyapatite," Acta Biomaterialia, 2006, vol. 2, pp. 201-206.

[12] B. M. Tracy and R. H. Doremus, "Direct electron microscopy studies of the bone-hydroxylapatite interface," J. Biomed. Mater. Res., 1984, vol.18, pp. 719-726.

[13] A.S. Posner and F. Betts, "Synthetic amorphous calcium phosphate and its relation to bone mineral structure," Accounts Chem. Res., 1975, vol. 8, pp. 273-281.

[14] Z. L. Raquel. Prog. Cryst. Growth. Ch., 1981, vol. 4, pp. 1-45.

[15] S. F. Hulbert, F. A. Young, R. S. Mathews, J. J. Klawitter, C. D. Talbert and F. H. Stelling, "Potential of ceramic materials as permanently implantable skeletal prostheses," J. Biomed. Mater. Res., 1970, vol. 4, pp. 433-456.

[16] Y. Phanny, "Development and characterization of polymer/bioceramic composite porous biomaterials for bone tissue engineering," PhD Thesis, Kyushu University, 2014.

[17] I.V. Yannas, "Regeneration of skin and nerve by use of collagen templates," In: Nimni, editor. Collagen-III. Boca Raton, FL: CRC Press, 1988. p. 87-115.

[18] D. Chow, M. L. Nunalee, D. W. Lim, A. J. Simnick, and A. Chilkoti, "Peptide-based biopolymers in biomedicine and biotechnology," Mater. Sci. Eng. R Rep., 2008, vol. 62, pp. 125-155.

[19] K. Paipitak, T. Pornpra, P. Mongkontalang, W. Techitdheera, and W. Pecharapa, "Characterization of PVA-Chitosan nanofibers prepared by electrospinning," Procedia Engineering, 2011, vol. 8, pp.101-105.

[20] WHO Scientific Group. World Health Organ Tech Rep Ser 919, 2003, 1 .

[21] I. E. Freed and G. Vunjak-novakovic, "Culture of organized cell communities," Advanced Drug Delivery Reviews, 1998, vol. 33 , pp. 15-30.

[22] M. E. Gomes, A. Salgado, and R. L. Reis, "Bone tissue engineering using starch based scaffolds obtained by different methods," In: REIS, R. \& COHN, D. (eds.) Polymer Based Systems on Tissue Engineering, Replacement and Regeneration, 2002, Springer Netherlands.

[23] M. S. Islam, Y. Kusumoto, and M. Abdulla-Al-Mamun, "Novel rose-type magnetic $\left(\mathrm{Fe}_{3} \mathrm{O}_{4}, \gamma-\mathrm{Fe}_{2} \mathrm{O}_{3}\right.$ and $\left.\alpha-\mathrm{Fe}_{2} \mathrm{O}_{3}\right)$ nanoplates synthesized by simple hydrothermal decomposition," M. Mater. Lett., 2011, vol. 66, pp. 165-167.

[24] T. Arahira and M. Todo, "Effects of proliferation and differentiation of mesenchymal stem cells on compressive mechanical behavior of collagen/ $\beta$-tcp composite scaffold," J. Mechan. Behav. Biomed. Mater., 2014, vol. 39, pp. 218-230.

[25] L. Sang, D. Luo, S. Xu, X. Wang, and X. Li, "Fabricationand evaluation of biomimetic scaffolds by using collagen-alginate fibrillargels for potential tissue engineering applications," Mater. Sci. Eng. C, 2011, vol. 31, pp. 262-271. 
[26] E. Landi, A. Tampieri, G. Celotti, and S. Sprio, "Densification behaviour and mechanisms of synthetic hydroxyapatites," J. Eur. Ceram. Soc., 2000, vol. 20, pp. 2377-2387.

[27] M. L. Munar, K. I. Udoh, K. Ishikawa, S. Matsuya, and M. Nakagawa, "Effects of sintering temperature over $1,300^{\circ} \mathrm{C}$ on the physical and compositional properties of porous hydroxyapatite foam,” Dent. Mater. J., 2006, Vol. 25, pp. $51-58$.
[28] N. Shanmugasundaram, P. Ravichandran, P. N. Reddy, N. Ramamurty, S. Pal, and K. P. Rao, "Collagen-chitosan polymeric scaffolds for the in vitro culture of human epidermoid carcinoma cells," Biomaterials, 2001, vol. 22, pp. 1943-1951. 